\section{KOMPASS}

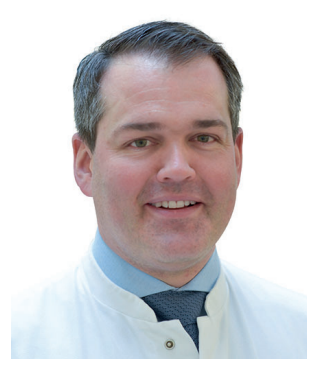

\section{Sebastian Stintzing}

Medizinische Klinik mit Schwerpunkt

Onkologie und Hämatologie (CCM),

Charité Universitätsmedizin Berlin, Berlin,

Deutschland

Spätestens seit der Veröffentlichung der aktuell gültigen S3-Leitlinie [1] sollte die Therapie des metastasierten kolorektalen Karzinoms (mKRK) durch die Ergebnisse der molekularen Pathologie geleitet werden. Neben der RAS Mutationstestung wird dabei auch die Untersuchung auf hohe Mikrosatelliteninstabilität (MSI-H) und BRAF V600E-Mutation als essenziell angesehen. Neben dem Allgemeinzustand der Patienten ermöglichen diese molekularen Analysen die Auswahl der besten Behandlungsoptionen im Rahmen der Erstlinientherapie. Bei den heutzutage erreichten Überlebenszeiten erhält dabei die individuelle Therapiestrategie und Therapiesequenz mit Erhaltungstherapien, Therapiepausen und Rechallenge eine zunehmende Relevanz und wird daher in aktuellen Studien untersucht.

\section{Adjuvante Therapieführung}

Nach den großen Adjuvanzstudien Anfang der 2000er Jahre war im UICC-Stadium III eine adjuvante Therapie bestehend aus 5-Fluorouracil (5-FU) und Oxaliplatin (FOLFOX) bzw. Capecitabin und Oxaliplatin über einen Zeitraum von 6 Monaten gesetzt [2]. Die Versuche, diese Therapie durch Antikörpergabe (anti-VEGF oder anti-EGFR) $[3,4]$ zu erweitern, scheiterten ebenso wie die Therapieerweiterung durch die Gabe von Irinotecan [5]. Erst durch die in 2017 veröffentlichte IDEA-Studie, in der bei mehr als 12800 Patienten die Nichtunterlegenheit

\title{
Darmkrebs - Aktuelle Standards und Perspektiven in der Therapieführung
}

der Therapiedauer von 3 Monaten gegen eine Therapiedauer von 6 Monaten getestet wurde, führte zu einem differenzierteren Ansatz in der adjuvanten Therapie [6]. Bei Patienten mit einem pT1-pT3 und pN1 Tumor kann die Therapie auf 3 Monate verkürzt werden. Hier sollte präferenziell die Kombination aus Capecitabin und Oxaliplatin (CAPOX) zum Einsatz kommen. Patienten mit einem hohen Rezidivrisiko (pT4 oder pN2) sollten weiterhin die adjuvante Therapie über eine Dauer von 6 Monaten erhalten, wenn aufgrund der Toxizität möglich. Aktuelle Studien im Stadium UICC III untersuchen unter anderem die Wirksamkeit von Immun-Checkpoint-Inhibitoren bei MSI-H Tumoren (ATOMIC Studie) sowie die Rolle von zirkulierender Tumor-DNA (ctDNA) als Risikomarker in der Entscheidungsfindung für oder gegen eine adjuvante Therapie bei Patienten mit UICC II Tumoren (CIRCULATE Studie). Auch in der adjuvanten Therapie kommt es also zu einer weiteren Individualisierung der Therapieoptionen.

\section{Palliative Therapie}

Im Rahmen der Erstlinientherapie wurden in den letzten Jahren insbesondere bei der Gruppe der RAS-Wildtyp (RASwt)-Tumoren Fortschritte in der Therapie und Patientenselektion erreicht. Die aktuelle S3-Leitlinie kolorektales Karzinom und auch die aktuelle ESMO Leitlinie nehmen klar Stellung: Tumoren, welche aus dem linken Kolon entstam- men und RASwt sind, sollten im Rahmen der Erstlinientherapie, wenn möglich, mit einer Kombination aus einer chemotherapeutischen Doublette (FOLFOX oder FOLFIRI) und einem EGFR-Antikörper behandelt werden $[1,7]$. Für die rechtsseitigen Tumoren gibt es aufgrund kleinerer Fallzahlen kein klares Bild. Hier zeigen in gepoolten Analysen RASwt Tumoren ein im Trend höheres Ansprechen unter EGFR-Antikörpertherapie. Bezüglich des progressionsfreien Überlebens (PFS) und Gesamtüberlebens (OS) ist die Kombination mit einem VEGF-Antikörper tendenziell besser [8]. Beide Therapieoptionen sind zugelassen und können verwendet werden. RAS-mutierte Tumoren (RASmt) sollten mit einer Doublette mit oder ohne VEGF-Antikörper behandelt werden.

Interessante Daten werden dieses Jahr noch für die Subgruppe der BRAF-mutierten Tumoren erwartet. In der Erstlinientherapie wird aufgrund einer retrospektiven Auswertung der italienischen TRIBE Studie die Gabe von FOLFOXIRI plus VEGF-Antikörper favorisiert [9]. Die Datenlage hierzu ist allerdings sehr begrenzt $(n=28)$. Eine bessere Entscheidungsgrundlage wird nach Abschluss der aktuell rekrutierenden FIRE4.5 Studie (AIO KRK-0116, Phase II Studie, $\mathrm{n}=99$ ) erwartet [10]. Hierbei wird FOLFOXIRI plus Cetuximab und FOLFOXIRI plus Bevacizumab an Patienten mit BRAFmutierten Tumoren verglichen.

Im Rahmen der Zweitlinientherapie wird die Wirksamkeit der chemotherapiefreien

\section{KARGER}

Fax +4976145207 14

information@karger.com

www.karger.com
() 2019 S. Karger GmbH, Freiburg

Accessible online at: www.karger.com/kko
Prof. Dr. Sebastian Stintzing

Medizinische Klinik mit Schwerpunkt Onkologie und Hämatologie (CCM)

Charité Universitätsmedizin Berlin

Charitéplatz 1, 10117 Berlin, Deutschland

Sebastian.stintzing@charite.de 
Kombination aus BRAF-Inhibitor, EGFR-Antikörper und MEK-Inhibitor getestet. Eine Phase II Studie (NCT01750918) zeigte hierbei gute Wirksamkeit der Dreifachkombination aus Panitumumab, Dabrafenib und Trametinib mit einem im Trend besseren Ansprechen und PFS für die Dreifachkombination [11]. Entscheidend für dieses Konzept werden die Daten der abgeschlossenen BEACON Studie (Phase III) sein, welche in 2019 erwartet werden. Die Auswertung der ersten 30 Patienten zeigten ein hohes Therapieansprechen bei einem medianen PFS der Zweitlinie von 8 Monaten für die Triplet Inhibition [12].

Die Therapieeskalation mit FOLFOXIRI ist ein Aspekt, der insbesondere durch die Daten der TRIBE Studie [13] sowie der kürzlich vorgestellten TRIBE-2 Studie und der aktuell veröffentlichten STEAM Studie weitere Diskussion erfährt. Die TRIBE-2 Studie testete die Gabe von FOLFOXIRI und Bevacizumab in der Induktionstherapie (8 Zyklen) und in der Reinduktion nach erster Progression gegenüber der sequentiellen Gabe von FOLFOX Bevacizumab und FOLFIRI Bevacizumab nach erster Progression. Der primäre Endpunkt (PFS2, Zeit bis zur zweiten Progression) wurde dabei erreicht und zeigte einen signifikanten Vorteil für die Induktionstherapie mit FOLFOXIRI (16,2 Monate versus 18,9 Monate; $p<0,001$; HR 0,69) [14]. Innerhalb von neuen Therapiestrategien spielen die Möglichkeiten der Wiederverwendung eines bereits erfolgreich getesteten Regimes im Rahmen sogenannter Rechallenge Therapien eine zunehmende Rolle. Hierbei werden vor allem die neuen diagnostischen Möglichkeiten der Flüssigbiopsie («Liquid biopsy») hinsichtlich ihres Einflusses auf die klinischen Entscheidungen untersucht. Die Tumorheterogenität, zu der auch in diesem Heft ein Artikel vorhanden ist, wird hier zunehmend Bedeutung erlangen. Für die MSI-H Subgruppe von mKRK Tumoren (etwa 5\%) sind mit den ImmunCheckpoint-Inhibitoren in den letzten Jahren Substanzen in die Klinik gekommen, welche zum Teil erstaunliche Wirkungen zeigen.
Hierzu findet sich auch ein Artikel in diesem Heft, der noch einmal hervorhebt, alle Tumoren frühzeitig auf MSI-H testen zu lassen.

Aktuell noch ausstehend sind die Daten der Keynote-177 Studie, welche Pembrolizumab gegenüber einer vom Arzt gewählten Standardtherapie bei MSI-H Tumoren im Rahmen der Erstlinientherapie vergleicht. Es wird erwartet, dass diese Daten zur Veränderung der Zulassung von Pembrolizumab im mKRK führen werden. Bis dahin muss beim mKRK vor Gabe eines Immun-Checkpoint-Inhibitors noch die Kostenzusage der Kostenträger eingeholt werden.

Als neue Therapieoption wird in diesem Jahr auch noch die Zulassung eines NTRK Inhibitors erwartet. NTRK-Fusionen sind innerhalb von kolorektalen Karzinomen sehr seltene Veränderungen (0,2$0,5 \%)$, können aber mit NTRK Inhibitoren sehr gut behandelt werden [15].

\section{Zusammenfassung}

Die Therapie des metastasierten kolorektalen Karzinoms differenziert sich weiter. Für kleine Subgruppen, wie MSI-H oder NTRK, ergeben sich neue Therapieoptionen. Die Seltenheit solcher Veränderungen stellt eine große Herausforderung dar, da momentan noch unklar ist, welcher Patient in welchem Krankheitsstadium auf diese Alterationen getestet werden sollte. Bereits heute gilt aber, dass alle Patienten mit mKRK noch vor Beginn einer Therapie auf RAS, BRAF und MSI-H getestet werden sollten, um die effektivste Therapiestrategie planen zu können.

Eine gute Zeit wünscht Ihnen

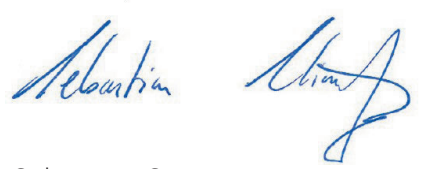

Sebastian Stintzing

\section{Literatur}

1 Schmiegel, W. and C. Pox, S3-Leitlinie Kolorektales Karzinom - Langversion 2.1. - Januar 2019, in AWMF-Registernummer: 021/007OL. 2019, AWMF: https://www.awmf.org/uploads/ tx_szleitlinien/021-007OLl_S3_KolorektalesKarzinom-KRK_2019-01.pdf (letzter Zugriff 30.04.2019)

-2 Andre, T., et al., Oxaliplatin, fluorouracil, and leucovorin as adjuvant treatment for colon cancer. N Engl J Med, 2004;350:2343-51.

-3 Taieb, J., et al., Oxaliplatin, fluorouracil, and leucovorin with or without cetuximab in patients with resected stage III colon cancer (PETACC-8): an open-label, randomised phase 3 trial. Lancet Oncol, 2014;15:862-73.

$\checkmark 4$ Allegra, C.J., et al., Phase III trial assessing bevacizumab in stages II and III carcinoma of the colon: results of NSABP protocol C-08. J Clin Oncol 2011;29:11-6.

5 Van Cutsem, E., et al., Randomized phase III trial comparing biweekly infusional fluorouracil/leucovorin alone or with irinotecan in the adjuvant treatment of stage III colon cancer: PETACC-3. J Clin Oncol 2009;27:3117-25.
6 Grothey, A., et al., Duration of Adjuvant Chemotherapy for Stage III Colon Cancer. N Engl J Med 2018;378:1177-1188.

7 Yoshino, T., et al., Pan-Asian adapted ESMO consensus guidelines for the management of patients with metastatic colorectal cancer: a JSMO-ESMO initiative endorsed by CSCO, KACO, MOS, SSO and TOS. Ann Oncol 2018; 29:44-70.

$>8$ Arnold, D., et al., Prognostic and predictive value of primary tumour side in patients with RAS wild-type metastatic colorectal cancer treated with chemotherapy and EGFR directed antibodies in six randomized trials. Ann Oncol 2017;28:1713-1729.

-9 Cremolini, C., et al., FOLFOXIRI plus bevacizumab versus FOLFIRI plus bevacizumab as first-line treatment of patients with metastatic colorectal cancer: updated overall survival and molecular subgroup analyses of the open-label, phase 3 TRIBE study. Lancet Oncol 2015.
10 Stintzing, S., FIRE-4.5-Studie. Forum 2017;32: 54. doi:10.1007/s12312-016-0186-6, 2017.

11 Corcoran, R.B., et al., Combined BRAF, EGFR, and MEK Inhibition in Patients with BRAF(V600E)-Mutant Colorectal Cancer. Cancer Discov 2018;8:428-443.

12 Van Cutsem, E., et al., Binimetinib, Encorafenib, and Cetuximab Triplet Therapy for Patients With BRAF V600E-Mutant Metastatic Colorectal Cancer: Safety Lead-In Results From the Phase III BEACON Colorectal Cancer Study. J Clin Oncol 2019;JCO1802459.

13 Loupakis, F., et al., Initial therapy with FOLFOXIRI and bevacizumab for metastatic colorectal cancer. N Engl J Med 2014;371: 1609-18.

14 Cremolini, C., et al., TRIBE2: a phase III, randomized strategy study by GONO in the 1stand 2nd-line treatment of unresectable metastatic colorectal cancer (mCRC) patients. 2018. ESMO Congress 2018.

15 Drilon, A., et al., Efficacy of Larotrectinib in TRK Fusion-Positive Cancers in Adults and Children. N Engl J Med 2018;378:731-739. 